This item was submitted to Loughborough's Research Repository by the author.

Items in Figshare are protected by copyright, with all rights reserved, unless otherwise indicated.

\title{
A note about integrable systems on low-dimensional Lie groups and Lie algebras
}

PLEASE CITE THE PUBLISHED VERSION

https://doi.org/10.1134/S156035471903002X

\section{PUBLISHER}

(C) Pleiades Publishing, Ltd.

\section{VERSION}

AM (Accepted Manuscript)

\section{PUBLISHER STATEMENT}

This work is made available according to the conditions of the Creative Commons Attribution-NonCommercialNoDerivatives 4.0 International (CC BY-NC-ND 4.0) licence. Full details of this licence are available at: https://creativecommons.org/licenses/by-nc-nd/4.0/

\section{LICENCE}

CC BY-NC-ND 4.0

\section{REPOSITORY RECORD}

Bolsinov, Alexey V., and Jinrong Bao. 2019. "A Note About Integrable Systems on Low-dimensional Lie Groups and Lie Algebras”. figshare. https://hdl.handle.net/2134/38371. 


\title{
A note about integrable systems on low dimensional Lie groups and Lie algebras
}

\author{
Alexey Bolsinov*and Jinrong Bao ${ }^{\dagger}$
}

\section{Contents}

1 Introduction

2 Integrability of left-invariant systems on Lie groups with 2-dimensional coadjoint orbits

3 Classification of left-invariant Riemannian and sub-Riemannian metrics on three dimensional Lie groups

4 Explicit description of left-invariant geodesic flows on non-semisimple 3-dimensional Lie groups

$5 \quad$ Example: the group $G_{\mathrm{VII}_{0}}=\widetilde{E(2)} \quad 14$

$\begin{array}{ll}\text { References } & 17\end{array}$

\section{Introduction}

This paper was motivated by the following natural question: are there any natural analogs of integrable cases in rigid body dynamics (e.g. Euler, Lagrange and Kovalevskaya tops) if one replaces the rotation group $S O(3)$ with another 3-dimensional group?

We do not discuss this question here (see [10] for recent progress in this direction) and the present paper can be considered as a preparatory work for further study of integrable cases on low-dimensional Lie groups. Our first goal is to explain why any left-invariant Hamiltonian system on (the cotangent bundle of) a 3-dimensonal Lie group $G$ (for instance, geodesic flows of Riemannian and sub-Riemannian metrics) is Liouville integrable. We show that integrability of such systems easily follows from the fact that the coadjoint orbits of $G$ are two-dimensional (Theorem 1) so that the dimension of $G$ and other properties, e.g., unimodularity (cf. [20]) are less important. Notice that if coadjoint orbits of $G$ have

\footnotetext{
${ }^{*}$ School of Mathematics, Loughborough Uniersity, LE11 3TU, UK and Faculty of Mechanics and Mathematics, Moscow State University, 11992, Russia, A.Bolsinov@lboro.ac.uk

${ }^{\dagger}$ School of Mathematics, Loughborough Uniersity, LE11 3TU, UK, J.Bao@lboro.ac.uk
} 
dimension $\geqslant 4$, it may well happen that $G$ admits no integrable left-invariant geodesic flows at all $[5,16,17,18]$.

Next we give normal forms of left-invariant Riemannian and sub-Riemannian metrics on 3-dimensional Lie groups. Recall that in general a left-invariant metric on a Lie group of dimension 3 is defined by six constants $g_{i j}=g_{j i}, i, j=1,2,3$, but some of them "can be killed" by means of the automorphism group. For instance, in the case of $S O(3)$ every leftinvariant metric (equivalently, the inertia tensor) can be reduced to a diagonal form so that there are only three essential parameters known as principal moments of inertia. We will provide a similar reduction for all simply connected Lie groups of dimension 3 (Theorem $3)$. We will focus, however, on the case of solvable groups as the cases of $S O(3)$ and $S L(2)$ have been already extensively studied: the rotation group $S O(3)$ leading to classical Euler and Euler-Poisson equations is a fundamental object in Geometry, Mechanics and Mathematical Physics [1, 9, 28], for $S L(2)$ we refer to recent papers [3, 19, 21, 22]. Our description is explicit and will be given in global coordinates on $G$ (Theorem 5).

We do not want to say that these results are essentially new. Geodesic flows of Lie groups is a very popular subject (in dimension 3 , see $[2,4,6,11,12,19,20]$ ) and the integrability mechanisms are now well understood (see e.g., [7, 8, 23]. We especially would like to refer to the paper [2] by Barrett et al., devoted to classification of left-invariant sub-Riemannian metrics on 3-dimensional Lie groups. The authors obtain classification in different terms which makes the comparison of their results with ours a non-obvious task. We definitely prefer our approach as we present the answer in a short explicit form (Theorems 3 and 5) that seems to be quite suitable for further studies of integrable systems on these Lie groups as illustrated in Section 5.

To avoid possible misunderstanding and confusion with the notation we are using below, we would like to emphasise that throughout the paper we identify the following objects related to Lie groups and Lie algebras:

- left-invariant vector fields on a Lie group $G$;

- left-invariant functions on the cotangent bundle $T^{*} G$;

- elements of the Lie algebra $\mathfrak{g}$ of the Lie group $G$;

- linear functions on the dual space $\mathfrak{g}^{*}$.

Notice that each of the corresponding vector spaces carries a natural structure of a Lie algebra (w.r.t. the Lie bracket of vector field, the canonical Poisson bracket on $T^{*} G$, the commutator on $\mathfrak{g}$ and the Lie-Poisson bracket on $\mathfrak{g}^{*}$ respectively). These Lie algebras are canonically isomorphic. For example, by $f_{0}, X_{1}, \ldots, X_{n-1}$ we denote a basis of leftinvariant vector fields on $G$, but we equally may think of them as a basis of the Lie algebra $\mathfrak{g}$, or Cartesian coordinates on $\mathfrak{g}^{*}$, or linear functions on $T^{*} G$.

This work was supported by the Russian Science Foundation (project no. 17-11-01303). The authors are very grateful to Alexey Borisov and Ivan Mamaev for valuable comments and stimulating discussions. 


\section{Integrability of left-invariant systems on Lie groups with 2-dimensional coadjoint orbits}

The purpose of this section is the following

Theorem 1. Let $G$ be a connected Lie group such that its (generic) coadjoint orbits are two-dimensional. Then any left-invariant Hamiltonian system on $T^{*} G$ is Liouville integrable, i.e., possesses $n=\operatorname{dim} G$ independent commuting integrals $F_{0}=H, F_{1}, \ldots, F_{n-1}$ Moreover, $F_{1}, \ldots, F_{n-1}$ are polynomial in momenta.

In particular, if $G$ is a three-dimensional Lie group, then every left-invariant Hamiltonian system on $T^{*} M$ is Liouville integrable.

Proof. The proof is based on the description of Lie groups $G$ satisfying the above condition obtained by A.Konyaev [15] and the classical Noether theorem that states the following:

Theorem 2 (Noether theorem). Consider a Hamiltonian system on $T^{*} M$ with a Hamiltonian $H$ and let $\xi$ be a vector field on $M$ that preserves the Hamiltonian ${ }^{1}$. Then $\xi$, as a linear function on $T^{*} M$, is a first integral of this Hamiltonian system, called Noether integral, i.e., $\{\xi, H\}=0$.

Let $H$ be an arbitrary left-invariant function on $T^{*} G$ and

$$
\dot{q}=\frac{\partial H}{\partial p}, \quad \dot{p}=-\frac{\partial H}{\partial q} .
$$

be the corresponding Hamiltonian system on $T^{*} G$ endowed with the canonical Poisson structure.

Recall the following simple fact: if $Y$ is a right-invariant vector field on $G$, then its flow $\Phi_{Y}^{t}: G \rightarrow G$ is given by

$$
\Phi_{Y}^{t}(x)=\exp \left(t Y_{e}\right) \cdot x=L_{\exp \left(t Y_{e}\right)}(x), \quad x \in G,
$$

where $Y_{e}=Y(e) \in T_{e} G$ is the the value of $Y$ at the identity element $e \in G$. In other words, the flow of a right-invariant vector field is given by left translations. In the context of the Noether theorem this means that left-invariant objects are preserved by right-invariant vector fields.

Let $Y_{1}, \ldots, Y_{n}$ denote a basis of right-invariant vector fields on $G$. In view of the above remark, $Y_{i}$ preserves $H$ and, according to the Noether theorem, $Y_{1}, \ldots, Y_{n}$ span an algebra $\mathcal{F}_{\text {right }}$ of (right-invariant) first integrals for the Hamiltonian system (1) (recall that we consider $Y_{i}$ as a function on $T^{*} G$ linear in momenta, i.e., we set $\left.Y_{i}(q, p)=\left\langle p, Y_{i}(q)\right\rangle\right)$. This property is, basically, equivalent to the fact that every left-invariant vector field commute with every rigth-invariant vector field on a Lie group $G$ and, similarly, every left-invariant function on $T^{*} G$ Poisson commute with every right-invariant function.

\footnotetext{
${ }^{1}$ This condition can be understood in the following way. Let $\Phi_{\xi}^{t}: M \rightarrow M$ be the (local) flow of $\xi$ on $M$. This map can be naturally lifted to the cotangent bundle $\widehat{\Phi}_{\xi}^{t}: T^{*} M \rightarrow T^{*} M$. Then " $\xi$ preserves $H$ " means $H\left(\widehat{\Phi}_{\xi}^{t}(q, p)\right)=H(q, p)$ for all $t \in \mathbb{R}$ and $(q, p) \in T^{*} M$. In other words, $H$ is preserved by the flow of $\xi$ naturally extended to $T^{*} M$.
} 
This Lie algebra $\mathcal{F}_{\text {right }}$ is obviously isomorphic to the Lie algebra $\mathfrak{g}$ of the Lie group $G$. In addition to that, the Hamiltonian $H$ itself is a first integral. To construct $n$ commuting integrals, we have to distinguish two different cases:

(i) $H$ is not right-invariant;

(ii) $H$ is right-invariant (that is, $H$ is in fact bi-invariant since by our assumption $H$ is left-invariant).

In the first case, $H$ is functionally independent of $Y_{1}, \ldots, Y_{n}$ and therefore can be added to the algebra of integrals so that we obtain a larger algebra of first integrals $\mathcal{F}=\mathcal{F}_{\text {right }} \oplus\langle H\rangle$.

In the second case, $H$ is a certain function of $Y_{1}, \ldots, Y_{n}$ and therefore cannot be considered as an essentially new first integral. However, if we take any left-invariant vector field $X$ which is not right invariant (such a vector field obviously exists as $G$ is not commutative), this vector field gives another Noether integral for our Hamiltonian system and the algebra of first integrals can be taken in the form $\mathcal{F}=\mathcal{F}_{\text {right }} \oplus\langle X\rangle$.

Thus, in the both cases we obtain a non-commutative finite-dimensional algebra of first integrals $\mathcal{F}$ that contains $n+1$ independent integrals. Moreover, from the algebraic viewpoint $\mathcal{F}$ is isomorphic to the direct sum of $\mathfrak{g}$ and a one-dimensional commutative Lie algebra (generated by either $H$ or $X$ ), i.e. $\mathcal{F} \simeq \mathfrak{g} \oplus \mathbb{R}$.

Our first remark is that $\mathcal{F}$ provides the integrability of (1) in the non-commutative sense (see, Mischenko, Fomenko [24]) or, in a slightly different terminology, (1) is superintegrable. Indeed, if the generators of the (finite-dimensional) algebra $\mathcal{F}$ of first integrals are functionally independent, then the non-commutative integrability condition takes the form

$$
\operatorname{dim} G=n=\frac{1}{2}(\operatorname{dim} \mathcal{F}+\operatorname{ind} \mathcal{F})
$$

and in our case we have $\operatorname{dim} \mathcal{F}=\operatorname{dim} \mathfrak{g}+\operatorname{dim} \mathbb{R}=n+1$ and ind $\mathcal{F}=$ ind $\mathfrak{g}+$ ind $\mathbb{R}=$ $(n-2)+1=n-1$. (Recall that the index of $\mathfrak{g}$ is the codimension of a generic coadjoint orbit which equals two in our case so that ind $\mathfrak{g}=n-2$ ). In particular, this implies that the invariant isotropic integal submanifolds ${ }^{2}$ have dimension $n-1^{3}$.

Now to complete the proof it remains to construct $n-1$ independent commuting polynomials $F_{1}, \ldots, F_{n-1}$ in $Y_{1}, Y_{2}, \ldots, Y_{n}$. In the theory of integrable system on finitedimensional Lie algebras, such a collection of polynomials is known as a complete commutative set of polynomials on the dual space $\mathfrak{g}^{*}$. The number of independent polynomials in this set must, by definition, be equal to $\frac{1}{2}(\operatorname{dim} \mathfrak{g}+\operatorname{ind} \mathfrak{g})$. Mischenko and Fomenko conjectured in [25] that such a collection exists for any finite-dimensional Lie algebra $\mathfrak{g}$, and this conjecture was proved by Sadetov [27] in 2004. This remark basically completes the proof, as we obtain $n$ commuting integrals of the form $F_{1}, \ldots, F_{n-1}$ and either $H$ (in case (i)) or $X$ (in case (ii)). All of these integrals (except perhaps for $H$ ) are polynomial in momenta by construction.

\footnotetext{
${ }^{2}$ We cannot say "invariant tori" as they are not necessarily compact.

${ }^{3}$ For example, invariant integral surfaces for left-invariant metrics on $S O(3)$ are not three- but twodimensional.
} 
We want, however, to describe commuting polynomials $F_{1}, \ldots, F_{n-1}$ explicitly without referring to a rather non-trivial construction from [27]. To that end, we use the classification of Lie algebras with two-dimensional generic coadjoint orbits obtained by Konyaev $[15]$.

According to his classification there is an infinite series of such algebras that are semidirect sums of a one-dimensional Lie algebra and a commutative ideal of an arbitrary dimension (see a more detailed description below) and six "exceptional" Lie algebras listed below:

Case 1: 3-dimensional Lie algebra so(3) with relations

$$
\left[\mathrm{e}_{1}, \mathrm{e}_{2}\right]=\mathrm{e}_{3}, \quad\left[\mathrm{e}_{1}, \mathrm{e}_{3}\right]=-\mathrm{e}_{2}, \quad\left[\mathrm{e}_{2}, \mathrm{e}_{3}\right]=\mathrm{e}_{1} .
$$

Case 2: 3-dimensional Lie algebra $s l(2)$ with relations

$$
\left[\mathrm{e}_{1}, \mathrm{e}_{2}\right]=\mathrm{e}_{1}, \quad\left[\mathrm{e}_{1}, \mathrm{e}_{3}\right]=-2 \mathrm{e}_{2}, \quad\left[\mathrm{e}_{2}, \mathrm{e}_{3}\right]=\mathrm{e}_{3} .
$$

Case 3: 4-dimensional Lie algebra $A_{4,8}$ with relations ${ }^{4}$

$$
\left[\mathrm{e}_{2}, \mathrm{e}_{3}\right]=\mathrm{e}_{1}, \quad\left[\mathrm{e}_{2}, \mathrm{e}_{4}\right]=\mathrm{e}_{2}, \quad\left[\mathrm{e}_{3}, \mathrm{e}_{4}\right]=-\mathrm{e}_{3} .
$$

Case 4: 4-dimensional Lie algebra $A_{4,10}$ with relations

$$
\left[\mathrm{e}_{2}, \mathrm{e}_{3}\right]=\mathrm{e}_{1}, \quad\left[\mathrm{e}_{2}, \mathrm{e}_{4}\right]=-\mathrm{e}_{3}, \quad\left[\mathrm{e}_{3}, \mathrm{e}_{4}\right]=\mathrm{e}_{2} .
$$

Case 5: 5-dimensional Lie algebra $A_{5,3}$ with relations

$$
\left[\mathrm{e}_{3}, \mathrm{e}_{4}\right]=\mathrm{e}_{5}, \quad\left[\mathrm{e}_{3}, \mathrm{e}_{5}\right]=\mathrm{e}_{1}, \quad\left[\mathrm{e}_{4}, \mathrm{e}_{5}\right]=\mathrm{e}_{3} .
$$

Case 6: 6-dimensional Lie algebra $A_{6,3}$ with relations

$$
\left[\mathrm{e}_{1}, \mathrm{e}_{2}\right]=\mathrm{e}_{6}, \quad\left[\mathrm{e}_{1}, \mathrm{e}_{3}\right]=\mathrm{e}_{4}, \quad\left[\mathrm{e}_{2}, \mathrm{e}_{3}\right]=\mathrm{e}_{5} .
$$

First we consider the infinite series mentioned above. Each $n$-dimensional Lie algebra from this series admits the following $n$-dimensional matrix representation:

$$
\left(\begin{array}{cc}
x_{0} A & \bar{x} \\
0 \ldots 0 & 0
\end{array}\right), \quad \text { where } \bar{x}=\left(\begin{array}{c}
x_{1} \\
\vdots \\
x_{n-1}
\end{array}\right), x_{0}, x_{1}, \ldots, x_{n-1} \in \mathbb{R}
$$

and $A$ is a certain $(n-1) \times(n-1)$ matrix which determines the type of $\mathfrak{g}$. Two Lie algebras of this kind with different matrices $A_{1}$ and $A_{2}$ are isomorphic if $A_{1}$ and $A_{2}$ satisfy the relation $A_{2}=\lambda P A_{2} P^{-1}$ for some nonzero $\lambda \in \mathbb{R}$ and invertible matrix $P$. As already noticed, this Lie algebra is a semidirect product of the one-dimensional Lie

\footnotetext{
${ }^{4}$ The notation $A_{n, k}$ is taken from [26]. Here $n$ denotes the dimension of $\mathfrak{g}$ and $k$ the number of this Lie algebra in the list of $n$-dimensional Lie algebras given in [26], $n \leqslant 6$.
} 
(sub)algebra generated by $\left(\begin{array}{cc}x_{0} A & \overline{0} \\ 0 \ldots 0 & 0\end{array}\right)$ and the $(n-1)$-dimensional commutative ideal $\mathfrak{h}=\left\{\left(\begin{array}{cc}0_{n-1} & \bar{x} \\ 0 \ldots 0 & 0\end{array}\right)\right\}$

A complete set consisting of $n-1$ independent commuting polynomials for this Lie algebra is easy to construct: one can just take the linear functions corresponding to the natural basis $\mathrm{e}_{1}, \ldots, \mathrm{e}_{n-1}$ of this commutative ideal. In other words, the first integrals on $T^{*} G$ are linear functions of the form $F_{i}(q, p)=\left\langle p, Y_{i}(q)\right\rangle$, where $Y_{i}(q)$ is the right-invariant vector field on $G$ generated by one the basis vectors $\mathrm{e}_{1}, \ldots, \mathrm{e}_{n-1}$ of the commutative ideal $\mathfrak{h} \subset \mathfrak{g} \simeq T_{e} G=\operatorname{Lie}(G)$. This shows, in particular, that all additional integral in this case are in fact linear. In particular, this is the case for all solvable Lie algebras of dimension 3.

To complete the proof, we need to construct a complete set of commuting polynomials for the remaining six "exceptional" Lie algebras from Konyaev's list. Recall that this set should contain $\operatorname{dim} \mathfrak{g}-1$ polynomials in $e_{1}, \ldots, e_{n}$ (the basis of $\mathfrak{g}$ which was used above to define commutation relations). This can be done in several different ways (and of course was done by many authors). Below we present one of possible answers for each of these Lie algebras individually:

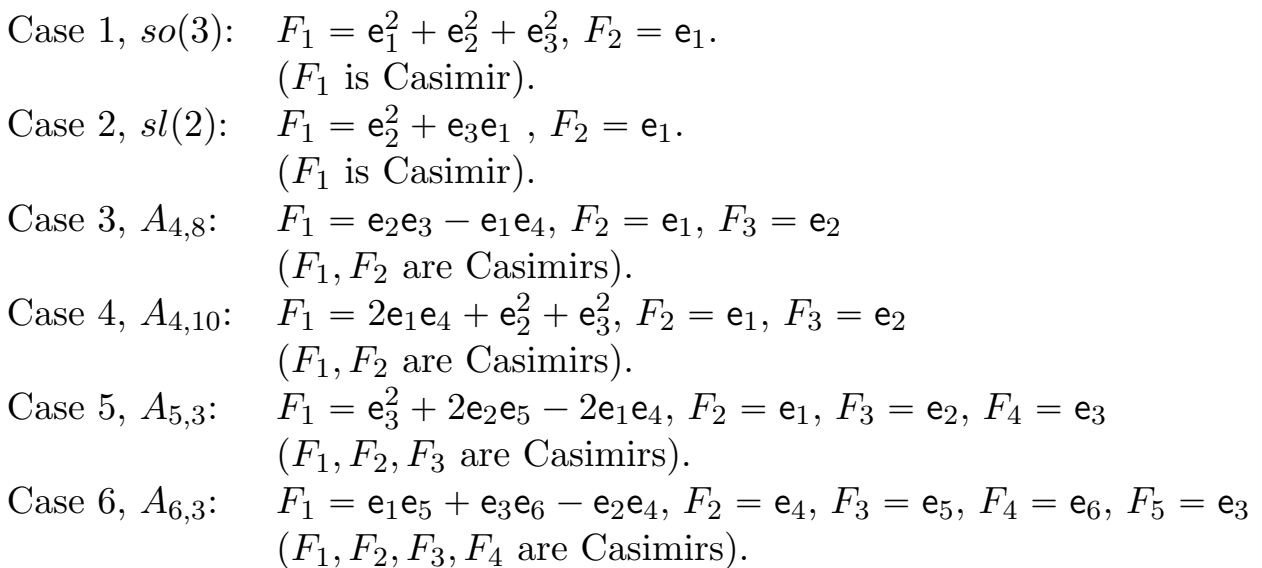

To get commuting functions $F_{1}, \ldots, F_{n-1}$ on $T^{*} G$ we just need to replace $\mathrm{e}_{i}$ in the above formulas by the corresponding linear function $Y_{i}(q, p)=\left\langle p, Y_{i}(q)\right\rangle$ where $Y_{i}$ denotes the right-invariant vector field on $G$ corresponding to the basis vector $\mathrm{e}_{i} \in \mathfrak{g}$.

\section{Classification of left-invariant Riemannian and sub-Riemannian metrics on three dimensional Lie groups}

Since we are working with both Riemannian and sub-Riemannian left-invariant metrics, it is more convenient to describe them in terms of the corresponding Hamiltonians on the cotangent bundle $T^{*} G$ (which can also be understood as quadratic forms on $T^{*} G$ ). Recall that for every left-invariant metric (Riemannian or sub-Riemannian) on a Lie group $G$, 
the corresponding Hamiltonian (quadratic form on $T^{*} G$ ) can be written as

$$
\frac{1}{2} \sum_{\alpha, \beta=1}^{n} g^{\alpha \beta} X_{\alpha} X_{\beta},
$$

where $X_{1}, \ldots, X_{n}(n=\operatorname{dim} G)$ is a basis of left-invariant vector fields on $G$ and $g^{\alpha \beta}$ are constants satisfying symmetry and (semi)-positive definiteness conditions.

Equivalently, $X_{1}, \ldots, X_{n}$ can be understood as the Cartesian coordinates on $\mathfrak{g}^{*}$ dual to a certain basis $e_{1}, \ldots, e_{n}$ of the Lie algebra $\mathfrak{g}$, and in fact by using the canonical identification of $\mathfrak{g}$ with $\left(\mathfrak{g}^{*}\right)^{*}$ we may assume that $\mathrm{e}_{i}=X_{i}$. In this view, a left-invariant metric on $G$ is defined by a quadratic form (with constant coefficients) on the dual space $\mathfrak{g}^{*}$. Our goal is to classify left-invariant metrics on $G$ (equivalently, positive (semi)-definite quadratic forms on $\mathfrak{g}^{*}$ ) up to the following natural equivalence relation.

Definition 1. Two quadratic forms (Hamiltonians)

$$
H=\frac{1}{2} \sum g^{\alpha \beta} X_{\alpha} X_{\beta} \quad \text { and } \quad \tilde{H}=\frac{1}{2} \sum \widetilde{g}^{\alpha \beta} X_{\alpha} X_{\beta}
$$

are said to be equivalent, if there is an automorphism $\phi: \mathfrak{g} \rightarrow \mathfrak{g}$ of the Lie algebra $\mathfrak{g}$ such that

$$
\frac{1}{2} \sum \tilde{g}^{\alpha \beta} X_{\alpha} X_{\beta}=\frac{1}{2} \sum g^{\alpha \beta} \phi\left(X_{\alpha}\right) \phi\left(X_{\beta}\right) .
$$

This equivalence relation guarantees that the corresponding automorphism $\Phi: G \rightarrow G$ is an isometry between the (sub-)Riemannian metrics corresponding to $H$ and $\tilde{H}$. In particular, the corresponding (canonical map) map $\Phi^{*}: T^{*} G \rightarrow T^{*} G$ transforms $H$ to $\tilde{H}$ so that the Hamiltonians $H$ and $\tilde{H}$ as well as the corresponding Hamiltonian systems are equivalent in the strongest possible sense.

Thus, for each 3-dimensional Lie group we just need to reduce a given $H=\frac{1}{2} \sum g^{\alpha \beta} X_{\alpha} X_{\beta}$ to a certain canonical form by means of transformations from the automorphism group $\operatorname{Aut}(\mathfrak{g})$ which is well known for each 3 -dimensional Lie algebra $\mathfrak{g}$. This can be done by elementary algebraic manipulations similar to those used in undergraduate Linear Algebra courses like "completing the square". We will demonstrate this procedure in detail for the algebra $\mathfrak{g}_{\mathrm{I}}$. Transferring it to all the other cases is just an easy exercise, but first we need to agree about the notation in order for the final result (Theorem 3) to make sense.

Below is the list of Lie algebras in dimension 3 (known as Bianchi classification) with a fixed basis $X_{0}, X_{1}, X_{2}$ and the automorphism group $\operatorname{Aut}(\mathfrak{g})$ explicitly written in terms of this basis ${ }^{5}$ (cf. [13, 14]).

For each Lie algebra we indicate non-trivial commutation relations between basis elements $X_{0}, X_{1}, X_{2}$ and then give an explicit matrix form $\mathrm{A}_{\phi}$ for the transformations $\phi: \mathfrak{g} \rightarrow \mathfrak{g}$ from the automorphism group Aut $(\mathfrak{g})$. The parameters $a, b, \alpha, \beta, \gamma, \delta$ in matrices $A_{\phi}$ below take arbitrary real values satisfying the only restriction that $\operatorname{det} A_{\phi} \neq 0$. In the cases of solvalble Lie algebras I-VII, $X_{0}, X_{1}$ and $X_{2}$ denote a basis of $\mathfrak{g}$ such that $X_{1}$ and $X_{2}$ generate a commutative ideal on which $X_{0}$ acts in a certain way.

\footnotetext{
${ }^{5}$ The full automorphism group could be slightly larger, but Aut(g) definitely contains its connected identity component, which is sufficient for our purposes.
} 
- $\mathfrak{g}_{1}: \quad$ commutative Lie algebra

$$
\left(\begin{array}{c}
X_{0}^{\prime} \\
X_{1}^{\prime} \\
X_{2}^{\prime}
\end{array}\right)=\mathrm{A}_{\phi}\left(\begin{array}{c}
X_{0} \\
X_{1} \\
X_{2}
\end{array}\right), \quad \mathrm{A}_{\phi} \in G L(3, \mathbb{R}), \quad \operatorname{dim} \operatorname{Aut}\left(\mathfrak{g}_{\mathfrak{\prime}}\right)=9
$$

- $\mathfrak{g}_{\|}$: (Heisenberg Lie algebra) $\left[X_{0}, X_{2}\right]=X_{1}$;

$$
\left(\begin{array}{l}
X_{0}^{\prime} \\
X_{1}^{\prime} \\
X_{2}^{\prime}
\end{array}\right)=\left(\begin{array}{ccc}
\alpha & a & \beta \\
0 & \alpha \delta-\gamma \beta & 0 \\
\gamma & b & \delta
\end{array}\right)\left(\begin{array}{l}
X_{0} \\
X_{1} \\
X_{2}
\end{array}\right), \quad \operatorname{dim} \operatorname{Aut}\left(\mathfrak{g}_{\mathrm{II}}\right)=6 .
$$

- $\mathfrak{g}_{\text {III }}: \quad\left[X_{0}, X_{1}\right]=X_{1}$;

$$
\left(\begin{array}{l}
X_{0}^{\prime} \\
X_{1}^{\prime} \\
X_{2}^{\prime}
\end{array}\right)=\left(\begin{array}{lll}
1 & a & b \\
0 & \alpha & 0 \\
0 & 0 & \beta
\end{array}\right)\left(\begin{array}{l}
X_{0} \\
X_{1} \\
X_{2}
\end{array}\right), \quad \operatorname{dim} \operatorname{Aut}\left(\mathfrak{g}_{\text {III }}\right)=4 .
$$

- $\mathfrak{g}_{\mathrm{I} \mathrm{V}:}:\left[X_{0}, X_{1}\right]=X_{1},\left[X_{0}, X_{2}\right]=X_{1}+X_{2}$;

$$
\left(\begin{array}{l}
X_{0}^{\prime} \\
X_{1}^{\prime} \\
X_{2}^{\prime}
\end{array}\right)=\left(\begin{array}{ccc}
1 & a & b \\
0 & \alpha & 0 \\
0 & \beta & \alpha
\end{array}\right)\left(\begin{array}{l}
X_{0} \\
X_{1} \\
X_{2}
\end{array}\right), \quad \operatorname{dim} \operatorname{Aut}\left(\mathfrak{g}_{\mathrm{g}} \mathrm{v}\right)=4 .
$$

- $\mathfrak{g} \mathrm{V}: \quad$ (book Lie algebra) $\left[X_{0}, X_{1}\right]=X_{1},\left[X_{0}, X_{2}\right]=X_{2}$;

$$
\left(\begin{array}{l}
X_{0}^{\prime} \\
X_{1}^{\prime} \\
X_{2}^{\prime}
\end{array}\right)=\left(\begin{array}{ccc}
1 & a & b \\
0 & \alpha & \beta \\
0 & \gamma & \delta
\end{array}\right)\left(\begin{array}{l}
X_{0} \\
X_{1} \\
X_{2}
\end{array}\right), \quad \operatorname{dim} \operatorname{Aut}\left(\mathfrak{g}_{\mathrm{v}}\right)=6
$$

- $\mathfrak{g}_{\mathrm{VI}_{0}}: \quad\left(\right.$ semidirect sum $\left.\mathrm{e}(1,1)=\mathrm{so}(1,1)+\mathbb{R}^{2}\right)\left[X_{0}, X_{1}\right]=X_{1},\left[X_{0}, X_{2}\right]=-X_{2}$;

$$
\left(\begin{array}{l}
X_{0}^{\prime} \\
X_{1}^{\prime} \\
X_{2}^{\prime}
\end{array}\right)=\left(\begin{array}{lll}
1 & a & b \\
0 & \alpha & 0 \\
0 & 0 & \beta
\end{array}\right)\left(\begin{array}{l}
X_{0} \\
X_{1} \\
X_{2}
\end{array}\right), \quad \operatorname{dim} \operatorname{Aut}\left(\mathfrak{g}_{\mathbf{v i}_{0}}\right)=4 .
$$

- $\mathfrak{g V l}_{\mathrm{V} !}:\left[X_{0}, X_{1}\right]=X_{1},\left[X_{0}, X_{2}\right]=a X_{2}$ with $a \neq \pm 1, a \neq 0$

$$
\left(\begin{array}{l}
X_{0}^{\prime} \\
X_{1}^{\prime} \\
X_{2}^{\prime}
\end{array}\right)=\left(\begin{array}{lll}
1 & a & b \\
0 & \alpha & 0 \\
0 & 0 & \beta
\end{array}\right)\left(\begin{array}{l}
X_{0} \\
X_{1} \\
X_{2}
\end{array}\right), \quad \operatorname{dim} \operatorname{Aut}\left(\mathfrak{g}_{\mathrm{v} \mathbf{l}}\right)=4 .
$$

- $\mathfrak{g V I I I}_{0}: \quad\left(\right.$ semidirect sum $\left.\mathrm{e}(2)=\mathrm{so}(2)+\mathbb{R}^{2}\right)\left[X_{0}, X_{1}\right]=-X_{2},\left[X_{0}, X_{2}\right]=X_{1}$;

$$
\left(\begin{array}{l}
X_{0}^{\prime} \\
X_{1}^{\prime} \\
X_{2}^{\prime}
\end{array}\right)=\left(\begin{array}{ccc}
1 & a & b \\
0 & \alpha & \beta \\
0 & -\beta & \alpha
\end{array}\right)\left(\begin{array}{l}
X_{0} \\
X_{1} \\
X_{2}
\end{array}\right), \quad \operatorname{dim} \operatorname{Aut}\left(\mathfrak{g}_{\mathrm{VII}_{0}}\right)=4 .
$$


- $\mathfrak{g}_{\mathrm{VII}}: \quad\left[X_{0}, X_{1}\right]=a X_{1}-X_{2},\left[X_{0}, X_{2}\right]=X_{1}+a X_{2}$.

$$
\left(\begin{array}{l}
X_{0}^{\prime} \\
X_{1}^{\prime} \\
X_{2}^{\prime}
\end{array}\right)=\left(\begin{array}{ccc}
1 & a & b \\
0 & \alpha & \beta \\
0 & -\beta & \alpha
\end{array}\right)\left(\begin{array}{l}
X_{0} \\
X_{1} \\
X_{2}
\end{array}\right), \quad \operatorname{dim} \operatorname{Aut}\left(\mathfrak{g}_{\mathrm{VII}}\right)=4
$$

- $\mathfrak{g}_{\mathrm{VIII}}: \quad($ simple Lie algebra $\operatorname{sl}(2, \mathbb{R}))\left[X_{0}, X_{1}\right]=2 X_{1},\left[X_{0}, X_{2}\right]=-2 X_{2},\left[X_{1}, X_{2}\right]=$ $X_{0}$.

$$
\left(\begin{array}{l}
X_{0}^{\prime} \\
X_{1}^{\prime} \\
X_{2}^{\prime}
\end{array}\right)=\mathrm{A}_{\phi}\left(\begin{array}{l}
X_{0} \\
X_{1} \\
X_{2}
\end{array}\right), \quad \mathrm{A}_{\phi} \in S O(2,1), \quad \operatorname{dim} \operatorname{Aut}\left(\mathfrak{g}_{\mathrm{V} I I I}\right)=3 .
$$

More specifically, $A_{\phi}^{\top}\left(\begin{array}{ccc}1 & 0 & 0 \\ 0 & 0 & 1 \\ 0 & 1 & 0\end{array}\right) A_{\phi}=\left(\begin{array}{lll}1 & 0 & 0 \\ 0 & 0 & 1 \\ 0 & 1 & 0\end{array}\right)$.

- $\mathfrak{g}_{\mathrm{IX}}: \quad($ simple Lie algebra $s o(3))\left[X_{0}, X_{1}\right]=X_{2},\left[X_{1}, X_{2}\right]=X_{0}\left[X_{2}, X_{0}\right]=X_{1}$.

$$
\left(\begin{array}{l}
X_{0}^{\prime} \\
X_{1}^{\prime} \\
X_{2}^{\prime}
\end{array}\right)=\mathrm{A}_{\phi}\left(\begin{array}{l}
X_{0} \\
X_{1} \\
X_{2}
\end{array}\right), \quad \mathrm{A}_{\phi} \in S O(3) \text {, i.e. } \mathrm{A}_{\phi}^{\top}=\mathrm{A}_{\phi}{ }^{-1}, \quad \operatorname{dim} \operatorname{Aut}\left(\mathfrak{g}_{\mid \mathrm{X}}\right)=3 .
$$

As a typical example, we now demonstrate the reduction-to-canonical-form process for the Lie algebra $\mathfrak{g}_{\mathfrak{l}}$. We start with an arbitrary quadratic form

$$
H=\sum_{i, j=0}^{2} g^{i j} X_{i} X_{j}
$$

Notice that due to the positive (semi-)definiteness of $H$, we have $g^{00}>0$. In the Riemannian case this fact is obvious. In the sub-Riemannian case, $g^{00}=0$ would imply that $H=g^{11} X_{1}^{2}+2 g^{12} X_{1} X_{2}+g^{22} X_{2}^{2}$ which is forbidden as $X_{1}$ and $X_{2}$ generate a proper ideal in $\mathfrak{g}_{\mathrm{I}}$ but not the whole Lie algebra. Thus, we can write

$$
H=g^{00}\left(X_{0}+\frac{g^{01}}{g^{00}} X_{1}+\frac{g^{02}}{g^{00}} X_{2}\right)^{2}+\text { quadratic form in } X_{1} \text { and } X_{2} \text {. }
$$

Since the transformation $X_{0}^{\prime}=X_{0}+\frac{g^{01}}{g^{00}} X_{1}+\frac{g^{02}}{g^{00}} X_{2}, X_{1}^{\prime}=X_{1}, X_{2}^{\prime}=X_{2}$ belongs to the automorphism group, we see that $H$ can be reduced to the form

$$
\tilde{H}=g^{00} X_{0}^{2}+a X_{1}^{2}+b X_{2}^{2}+2 c X_{1} X_{2}
$$

for some $a, b, c \in \mathbb{R}$.

Next for the same reason as above we notice that $b>0$ (in the sub-Riemannian case, $b=0$ would imply that $H=g^{00} X_{0}^{2}+a X_{1}^{2}$ which is forbidden as $X_{0}$ and $X_{1}$ generate a proper subalgebra of $\mathfrak{g}_{\mathrm{IV}}$ ). Hence, we can rewrite $\tilde{H}$ in the form

$$
\tilde{H}=g^{00} X_{0}^{2}+a X_{1}^{2}+b X_{2}^{2}+2 c X_{1} X_{2}=g^{00} X_{0}^{2}+\left(\sqrt{b} X_{2}+\frac{c}{\sqrt{b}} X_{1}\right)^{2}+\left(a-\frac{c^{2}}{b}\right) X_{1}^{2}
$$


Now applying the automorphism $X_{0}^{\prime}=X_{0}, X_{1}^{\prime}=\sqrt{b} X_{1}, X_{2}^{\prime}=\frac{c}{\sqrt{b}} X_{1}+\sqrt{b} X_{2}$, we conclude that $\tilde{H}$ is equivalent to

$$
g^{00} X_{0}^{2}+X_{2}^{2}+\left(\frac{a b-c^{2}}{b}\right) X_{1}^{2}
$$

or, by using simpler notation,

$$
A X_{0}^{2}+X_{2}^{2}+C X_{1}^{2}, \quad A, C>0 .
$$

In the sub-Riemannian case the last term in this expression has to automatically disappear and we come to the following conclusion:

Proposition 1. Every left-invariant Riemannian metric on the 3-dimensional Lie group $G_{\mathrm{IV}}$ is defined up to equivalence by the quadratic form

$$
H=A X_{0}^{2}+X_{2}^{2}+C X_{1}^{2}, \quad \text { for some } A, C>0 .
$$

Every left-invariant sub-Riemannian metric on the 3-dimensional Lie group $G_{\mathrm{IV}}$ is defined up to equivalence by the quadratic form

$$
H=A X_{0}^{2}+X_{2}^{2}, \quad \text { for some } A>0 .
$$

Similar elementary computations for all the other 3-dimensional Lie algebras lead to the following final result.

Let $G$ be a simply connected three-dimensional group and $X_{0}, X_{1}, X_{2}$ be a basis of left-invariant vector fields. This basis can be treated as a basis of the corresponding Lie algebra, one of those from Bianchi classification. In each case, we will assume that this basis is canonical, i.e. coincides with the basis described above for each algebra from the Bianchi list $\mathfrak{g}_{1}, \ldots, \mathfrak{g}_{\mathrm{VII}}$. As already discussed, it will be more convenient for us to define left-invariant metrics by means of the corresponding Hamiltonians being quadratic forms in $X_{0}, X_{1}, X_{2}$ with constant coefficients. In this setting we have

Theorem 3. The canonical forms of left-invariant Riemannian and sub-Riemannian metrics on simply connected three dimensional Lie groups are

Riemannian

$\begin{array}{ll}G_{\mathrm{I}} & X_{0}^{2}+X_{2}^{1}+X_{2}^{2} \\ G_{\mathrm{II}} & X_{0}^{2}+B X_{1}^{2}+X_{2}^{2} \\ G_{\mathrm{III}} & A X_{0}^{2}+X_{1}^{2}+X_{2}^{2}+C X_{1} X_{2} \\ G_{\mathrm{IV}} & A X_{0}^{2}+B X_{1}^{2}+X_{2}^{2} \\ G_{\mathrm{V}} & A X_{0}^{2}+X_{1}^{2}+X_{2}^{2} \\ G_{\mathrm{VI}} \text { and } G_{\mathrm{VI}_{0}} & A X_{0}^{2}+X_{1}^{2}+X_{2}^{2}+C X_{1} X_{2} \\ G_{\mathrm{VII}} \text { and } G_{\mathrm{VII}} & A X_{0}^{2}+B X_{1}^{2}+X_{2}^{2} \\ G_{\mathrm{VIII}} & A X_{0}^{2}+B\left(X_{1}^{2}+X_{2}^{2}\right)+C X_{1} X_{2} \\ & \\ G_{\mathrm{IX}} & A X_{0}^{2}+B X_{1}^{2}+C X_{2}^{2}\end{array}$

sub-Riemannian

none

$X_{0}^{2}+X_{2}^{2}$

$A X_{0}^{2}+\left(X_{1}+X_{2}\right)^{2}$

$A X_{0}^{2}+X_{2}^{2}$

none

$A X_{0}^{2}+\left(X_{1}+X_{2}\right)^{2}$

$A X_{0}^{2}+X_{2}^{2}$

$A X_{0}^{2}+B\left(X_{1}+X_{2}\right)^{2}$ or

$B\left(X_{1}^{2}+X_{2}^{2}\right)+C X_{1} X_{2}$

$A X_{0}^{2}+B X_{1}^{2}$ 
Here $A, B, C \in \mathbb{R}$ are arbitrary parameters satisfying the positive (semi-)definiteness assumption (more precisely $A>0$ and $B>0$ in all the cases, $|C|<2$ for $G_{\mathrm{III}}, G_{\mathrm{VI}}$ and $G_{\mathrm{VI}_{0}},|C|<2 B$ for $G_{\mathrm{VIII}}$, and $C>0$ for $\left.G_{\mathrm{IX}}\right)$.

The assumption that $G$ is simply connected can sometimes be important for the following reason. We consider the automorphism group Aut $(\mathfrak{g})$ and then extend its action to the group $G$. If $G$ is simply connected, then such an extension always exists. Otherwise, there might be some topological obstructions. In other words, the automorphism group of $G$ can be smaller than that of $\mathfrak{g}$. For example, if instead of the abelian group $\mathbb{R}^{3}$ we consider the torus $\mathbb{R}^{3} / \Gamma$ which is still an abelian Lie group with the same Lie algebra $\mathfrak{g}_{\mathrm{l}}$, then in order for an automorphism $\phi: \mathfrak{g}_{\mathbf{l}} \rightarrow \mathfrak{g}_{\mathbf{l}}$ to induce an automorphism $\Phi: \mathbb{R}^{3} / \Gamma \rightarrow \mathbb{R}^{3} / \Gamma$, we need an additional condition that $\phi$ preserves the lattice $\Gamma$.

In the next section we give explicit formulas for left- and right-invariant vector fields on solvable three-dimensional Lie groups in local coordinates. This will give us a possibility to study the corresponding geodesic flows from the analytic viewpoint and, in particular, to explicitly integrate them.

\section{Explicit description of left-invariant geodesic flows on non- semisimple 3-dimensional Lie groups}

Without loss of generality we may assume that the corresponding Lie group $G$ takes the following matrix form:

$$
G=G_{A}=\left\{\left(\begin{array}{ccc}
\exp \left(q_{0} A\right) & \bar{q} \\
0 \ldots & \ldots & 1
\end{array}\right), \quad \text { where } \bar{q}=\left(q_{1}, \ldots, q_{n-1}\right)^{\top} \in \mathbb{R}^{n-1}\right\} \subset G L(n, \mathbb{R}) .
$$

The parameters $q_{0}, q_{1}, \ldots, q_{n-1}$ are treated as global coordinates on the group. Topologically, this group is diffeomorphic to $\mathbb{R}^{n}$. Then we can just consider the coordinates $\left(q_{0}, \bar{x}\right)$ to study this group.

It is easy to prove that the multiplication in $G$ in these coordinates can be written as follows:

$$
\left(q_{0}, \bar{q}\right) *\left(y_{0}, \bar{y}\right)=\left(q_{0}+y_{0}, \exp \left(q_{0} A\right) \bar{y}+\bar{q}\right) .
$$

Each right- and left-invariant vector field is given by $n=\operatorname{dim} G$ arbitrary parameters. For right-invariant vector fields, we will denote them by $\eta^{0}, \eta^{1}, \ldots, \eta^{n-1}$, for left-invariant by $\xi^{0}, \xi^{1}, \ldots, \xi^{n-1}$. We now prove

Proposition 2. A left-invariant vector field $X_{\xi}$ on $G$ takes the form:

$$
X_{\left(\xi^{0}, \bar{\xi}\right)}=\left(\xi^{0}, \exp \left(q_{0} A\right) \bar{\xi}\right)=\xi^{0} \frac{\partial}{\partial q_{0}}+\sum_{i, j=1}^{n-1} B_{j}^{i}\left(q_{0}\right) \xi^{j} \frac{\partial}{\partial q_{i}}, \quad \text { where } \bar{\xi}=\left(\begin{array}{c}
\xi^{1} \\
\vdots \\
\xi^{n-1}
\end{array}\right)
$$


and $B_{j}^{i}\left(q_{0}\right)$ are the components of the matrix $\exp \left(q_{0} A\right)$. A right-invariant vector field $Y_{\eta}$ on $G$ takes the form

$$
Y_{\left(\eta^{0}, \bar{\eta}\right)}=\left(\eta^{0}, \eta^{0} \cdot A \bar{q}+\bar{\eta}\right)=\eta^{0} \frac{\partial}{\partial x^{0}}+\sum_{i, j=1}^{n-1}\left(\eta^{0} A_{j}^{i} q_{j}+\eta^{i}\right) \frac{\partial}{\partial x^{i}}, \quad \text { where } \bar{\eta}=\left(\begin{array}{c}
\eta^{1} \\
\vdots \\
\eta^{n-1}
\end{array}\right) .
$$

Proof. For verification we will use the following standard method for constructing left- and right-invariant vector fields on Lie groups. let $\gamma(t)$ be an arbitrary curve in $G$ such that $\gamma(0)=e$ (the identity of the group) and $\frac{d}{d t} \gamma(0)=\xi \in T_{e} G \simeq \mathfrak{g}$. Then the left-invariant vector field generated by $\xi$ is defined by the formula

$$
X_{\xi}(x)=\left.\frac{d}{d t}\right|_{t=0}(x * \gamma(t)), \quad x \in G .
$$

Similarly, for right-invariant vector fields: $Y_{\xi}=\left.\frac{d}{d t}\right|_{t=0}(\gamma(t) * x)$.

In our case, we set $\gamma(t)=\left(\xi^{0} t, \bar{\xi} t\right)$. Hence, by using formula (3):

$$
X_{\left(\xi^{0}, \bar{\xi}\right)}=\left.\frac{d}{d t}\right|_{t=0}\left(\left(q_{0}, \bar{q}\right) *\left(\xi^{0} t, \bar{\xi} t\right)\right)=\left.\frac{d}{d t}\right|_{t=0}\left(q_{0}+\xi_{0} t, \exp \left(q_{0} A\right) \bar{\xi} t+\bar{q}\right)=\left(\xi_{0}, \exp \left(q_{0} A\right) \bar{\xi}\right) .
$$

Similarly, for $\gamma(t)=\left(\eta^{0} t, \bar{\eta} t\right)$ we have

$$
Y_{\left(\eta^{0}, \bar{\eta}\right)}=\left.\frac{d}{d t}\right|_{t=0}\left(\left(\eta_{0} t, \bar{\eta} t\right) *\left(q_{0}, \bar{q}\right)\right)=\left.\frac{d}{d t}\right|_{t=0}\left(\eta_{0} t+q_{0}, \exp \left(\eta_{0} A t\right) \bar{q}+\bar{\eta} t\right)=\left(\eta_{0}, \eta_{0} A \bar{q}+\bar{\eta}\right),
$$

as stated.

Remark 1. Notice that the vector fields $\frac{\partial}{\partial q_{1}}, \ldots, \frac{\partial}{\partial q_{n-1}}$ are right-invariant. One more right-invariant vector field takes the form $\frac{\partial}{\partial x^{0}}+\sum_{i, j=1}^{n-1} A_{j}^{i} q_{j} \frac{\partial}{\partial x^{i}}$.

Remark 2. It is straightforward to check that left- and right-invariant vector fields given by (4) and (5) commute, i.e., $\left[X_{\left(\xi^{0}, \bar{\xi}\right)}, X_{\left(\eta^{0}, \bar{\eta}\right)}\right]=0$ for any $\left(\xi^{0}, \bar{\xi}\right)$ and $\left(\eta^{0}, \bar{\eta}\right)$.

Now if we choose an arbitrary basis in the space of left-invariant vector fields $X_{0}, \ldots, X_{n-1}$ then by treating them as linear functions on the cotangent bundle $T^{*} G$, we can have the corresponding Hamiltonian in terms of canonical coordinates $(x, p)$. Observe that the Hamiltonian $H$ does not contain variables $q_{1}, \ldots, q_{n-1}$ so that $p_{1}, \ldots, p_{n-1}$ are commuting integrals of the corresponding geodesic flow (which, of course, correspond to right-invariant vector fields $\frac{\partial}{\partial q_{1}}, \ldots, \frac{\partial}{\partial q_{n-1}}$. In particular, we are led to the following conclusion:

Theorem 4. The geodesic flow of any left-invariant Rienannian or sub-Riemannian metric on a Lie group $G$ defined by (2) is Liouville integrable. As commuting integrals, one can consider the momenta $p_{1}, \ldots, p_{n-1}$ and the Hamiltonian $H$ itself.

As we see, explicit formulas for left-invariant vector fields (as well as for left-invariant metrics) depend on the matrix $A$ that defines the group $G=G_{A}$. For the three-dimensional group of this type, these formulas are summarised in the following table (for each group 
we indicate the corresponding $2 \times 2$ matrix $A$ and its exponent $\exp \left(q_{0} A\right)$ used in the general formula (4) for left-invariant vector fields). Notice that the commutation relations between the left-invariant $X_{0}, X_{1}, X_{2}$ described below agree with those we used in Section 3 to introduce the list of non-semisimple Lie algebras $\mathfrak{g}_{\mathrm{l}}, \ldots, \mathfrak{g} \mathrm{VII}$.
Group $\quad A$
$\exp \left(q_{0} A\right)$
Basis of left-invaraint vector fields

$\begin{array}{lll}G_{\mid}: & \left(\begin{array}{ll}0 & 0 \\ 0 & 0\end{array}\right) & \left(\begin{array}{ll}1 & 0 \\ 0 & 1\end{array}\right) \\ G_{||}: & \left(\begin{array}{ll}0 & 1 \\ 0 & 0\end{array}\right) & \left(\begin{array}{cc}1 & q_{0} \\ 0 & 1\end{array}\right) \\ G_{\| \mid !}: & \left(\begin{array}{ll}1 & 0 \\ 0 & 0\end{array}\right) & \left(\begin{array}{cc}e^{q_{0}} & 0 \\ 0 & 1\end{array}\right)\end{array}$

$\left\{\begin{array}{l}X_{0}=\partial_{q_{0}} \\ X_{1}=\partial_{q_{1}} \\ X_{2}=\partial_{q_{2}}\end{array}\right.$

$G_{\mathrm{IV}}: \quad\left(\begin{array}{ll}1 & 1 \\ 0 & 1\end{array}\right) \quad\left(\begin{array}{cc}e^{q_{0}} & q_{0} e^{q_{0}} \\ 0 & e^{q_{0}}\end{array}\right)$

$\left\{\begin{array}{l}X_{0}=\partial_{q_{0}} \\ X_{1}=\partial_{q_{1}} \\ X_{2}=q_{0} \partial_{q_{1}}+\partial_{q_{2}}\end{array}\right.$

$\left(\begin{array}{ll}0 & 0\end{array}\right)\left(\begin{array}{ll}0 & 1\end{array}\right) \quad\left\{\begin{array}{l}X_{1}=e^{q_{0}} \partial_{q_{1}} \\ X_{2}=\partial_{q_{2}}\end{array}\right.$

$\left\{\begin{array}{l}X_{0}=\partial_{q_{0}} \\ X_{1}=e^{q_{0}} \partial_{q_{1}} \\ X_{2}=q_{0} e^{q_{0}} \partial_{q_{1}}+e^{q_{0}} \partial_{q_{2}}\end{array}\right.$

$G_{\mathrm{V}}: \quad\left(\begin{array}{ll}1 & 0 \\ 0 & 1\end{array}\right) \quad\left(\begin{array}{cc}e^{q_{0}} & 0 \\ 0 & e^{q_{0}}\end{array}\right)$

$\left\{\begin{array}{l}X_{0}=\partial_{q_{0}} \\ X_{1}=e^{q_{0}} \partial_{q_{1}} \\ X_{2}=e^{q_{0}} \partial_{q_{2}}\end{array}\right.$

$G_{\mathrm{VI}_{0}}: \quad\left(\begin{array}{cc}1 & 0 \\ 0 & -1\end{array}\right) \quad\left(\begin{array}{cc}e^{q_{0}} & 0 \\ 0 & e^{-q_{0}}\end{array}\right)$

$\left\{\begin{array}{l}X_{0}=\partial_{q_{0}} \\ X_{1}=e^{q_{0}} \partial_{q_{1}} \\ X_{2}=e^{-q_{0}} \partial_{q_{2}}\end{array}\right.$

$G_{\mathrm{VI}}: \quad\left(\begin{array}{cc}1 & 0 \\ 0 & a\end{array}\right) \quad\left(\begin{array}{cc}e^{q_{0}} & 0 \\ 0 & e^{a q_{0}}\end{array}\right)$

$\left\{\begin{array}{l}X_{0}=\partial_{q_{0}} \\ X_{1}=e^{q_{0}} \partial_{q_{1}} \\ X_{2}=e^{a q_{0}} \partial_{q_{2}}\end{array}\right.$

$G_{\mathrm{VII}_{0}}: \quad\left(\begin{array}{cc}0 & 1 \\ -1 & 0\end{array}\right) \quad\left(\begin{array}{cc}\cos q_{0} & \sin q_{0} \\ -\sin q_{0} & \cos q_{0}\end{array}\right)$

$\left\{\begin{array}{l}X_{0}=\partial_{q_{0}} \\ X_{1}=\cos q_{0} \partial_{q_{1}}-\sin q_{0} \partial_{q_{2}} \\ X_{2}=\sin q_{0} \partial_{q_{1}}+\cos q_{0} \partial_{q_{2}}\end{array}\right.$

$G_{\mathrm{VII}}: \quad\left(\begin{array}{cc}a & 1 \\ -1 & a\end{array}\right) \quad\left(\begin{array}{cc}e^{a q_{0}} \cos q_{0} & e^{a q_{0}} \sin q_{0} \\ -e^{a q_{0}} \sin q_{0} & e^{a q_{0}} \cos q_{0}\end{array}\right) \quad\left\{\begin{array}{l}X_{0}=\partial_{q_{0}} \\ X_{1}=e^{a q_{0}} \cos q_{0} \partial_{q_{1}}-e^{a q_{0}} \sin q_{0} \partial_{q_{2}} \\ X_{2}=e^{a q_{0}} \sin q_{0} \partial_{q_{1}}+e^{a q_{0}} \cos q_{0} \partial_{q_{2}}\end{array}\right.$

Combining these formulas with Theorem 3 we obtain

Theorem 5. The canonical forms of left-invariant Riemannian and sub-Riemannian metrics on solvable simply connected Lie groups of dimension 3 (in local coordinates $q_{0}, q_{1}, q_{2}$ introduced in (2)) are presented in the following table: 
Riemannian case

$G_{1}: \quad p_{0}^{2}+p_{1}^{2}+p_{2}^{2}$

$G_{\|}: \quad p_{0}^{2}+B p_{1}^{2}+\left(q_{0} p_{1}+p_{2}\right)^{2}$

$G_{\text {III }}: \quad A p_{0}^{2}+e^{2 q_{0}} p_{1}^{2}+p_{2}^{2}+C e^{q_{0}} p_{1} p_{2}$

$G_{\text {IV }}: \quad A p_{0}^{2}+e^{2 q_{0}}\left(B p_{1}^{2}+\left(q_{0} p_{1}+p_{2}\right)^{2}\right)$

$G_{\mathrm{V}}: \quad A p_{0}^{2}+e^{2 q_{0}}\left(p_{1}^{2}+p_{2}^{2}\right)$

$G_{\mathrm{VI}_{0}}: \quad A p_{0}^{2}+e^{2 q_{0}} p_{1}^{2}+e^{-2 q_{0}} p_{2}^{2}+C p_{1} p_{2}$

$G_{\mathrm{VI}}: \quad A p_{0}^{2}+e^{2 q_{0}} p_{1}^{2}+e^{2 a q_{0}} p_{2}^{2}+C e^{(a+1) q_{0}} p_{1} p_{2}$

$G_{\mathrm{VII}_{0}}: \quad A p_{0}^{2}+B\left(\cos q_{0} \cdot p_{1}-\sin q_{0} \cdot p_{2}\right)^{2}$ $+\left(\sin q_{0} \cdot p_{1}+\cos q_{0} \cdot p_{2}\right)^{2}$
sub-Riemannian case

none

$p_{0}^{2}+\left(q_{0} p_{1}+p_{2}\right)^{2}$

$A p_{0}^{2}+\left(e^{q_{0}} p_{1}+p_{2}\right)^{2}$

$A p_{0}^{2}+e^{2 q_{0}}\left(q_{0} p_{1}+p_{2}\right)^{2}$

none

$A p_{0}^{2}+\left(e^{q_{0}} p_{1}+e^{-q_{0}} p_{2}\right)^{2}$

$A p_{0}^{2}+\left(e^{q_{0}} p_{1}+e^{a q_{0}} p_{2}\right)^{2}$

$A p_{0}^{2}+\left(\sin q_{0} \cdot p_{1}+\cos q_{0} \cdot p_{2}\right)^{2}$

$\begin{aligned} G_{\mathrm{VII}}: & A p_{0}^{2}+e^{2 a q_{0}}\left(C\left(\cos q_{0} \cdot p_{1}-\sin q_{0} \cdot p_{2}\right)^{2} \quad A p_{0}^{2}+e^{2 a q_{0}}\left(\sin q_{0} \cdot p_{1}+\cos q_{0} \cdot p_{2}\right)^{2}\right. \\ & \left.+\left(\sin q_{0} \cdot p_{1}+\cos q_{0} \cdot p_{2}\right)^{2}\right)\end{aligned}$

Here $A, B, C \in \mathbb{R}$ are arbitrary parameters satisfying the positive (semi-)definiteness assumption (more precisely $A>0, B>0$ and $C^{2}<4$ ).

\section{Example: the group $G_{\mathrm{VII}_{0}}=\widetilde{E(2)}$}

Let us consider the geodesic flow for a left-invariant sub-Riemannian metric on $G_{\mathrm{VII}_{0}}$ in more detail.

The Hamiltonian takes the form $H=\frac{1}{2}\left(A p_{0}^{2}+\left(\sin q_{0} \cdot p_{1}+\cos q_{0} \cdot p_{2}\right)^{2}\right)$ and it admits three non-commuting linear integrals (that represent basis right-invariant vector fields, see Remark 1).

$$
\begin{aligned}
& F_{0}=p_{0}+q_{2} p_{1}-q_{1} p_{2}, \\
& F_{1}=p_{1}, \\
& F_{2}=p_{2} .
\end{aligned}
$$

Together with $H$, all these integrals form a four-dimensional (non-commutative) algebra of first integrals with two Casimir functions $H$ and $F=F_{1}^{2}+F_{2}^{2}$.

The corresponding sub-Riemannian geodesics can be easily found in quadratures. We are going to describe the geodesics through the identity of the group which is the origin of our coordinate system, i.e., $\left(q_{0}, q_{1}, q_{2}\right)=(0,0,0)$.

Indeed, let us fix the values of $F_{0}, F_{1}$ and $F_{2}$ :

$$
F_{0}=p_{0}+q_{2} p_{1}-q_{1} p_{2}=c_{0}, \quad F_{1}=p_{1}=c_{1}, \quad F_{2}=p_{2}=c_{2} .
$$


Then the Hamiltonian system with the Hamiltonian $H$ can be rewritten in variables $q_{1}, q_{2}$ and $q_{3}$ only:

$$
\begin{aligned}
& \frac{d q_{0}}{d t}=\frac{\partial H}{\partial p_{0}}=A p_{0}=A\left(c_{0}-q_{2} c_{1}-q_{1} c_{2}\right), \\
& \frac{d q_{1}}{d t}=\frac{\partial H}{\partial p_{1}}=\sin q_{0}\left(p_{1} \sin q_{0}+p_{2} \cos q_{0}\right)=\sin q_{0}\left(c_{1} \sin q_{0}+c_{2} \cos q_{0}\right), \\
& \frac{d q_{2}}{d t}=\frac{\partial H}{\partial p_{2}}=\cos q_{0}\left(p_{1} \sin q_{0}+p_{2} \cos q_{0}\right)=\cos q_{0}\left(c_{1} \sin q_{0}+c_{2} \cos q_{0}\right) .
\end{aligned}
$$

This dynamical system in $\mathbb{R}^{3}\left(q_{1}, q_{2}, q_{3}\right)$ now depends on $c_{0}, c_{1}, c_{2}$ as parameters and admits a non-trivial energy integral that can now be written as

$$
H=\frac{1}{2}\left(A\left(c_{0}-c_{1} q_{2}+c_{2} q_{1}\right)^{2}+\left(c_{1} \sin q_{0}+c_{2} \cos q_{0}\right)^{2}\right) .
$$

For simplicity, set $c_{0}=1, c_{1}=1, c_{2}=0$ (the general case is not essentially different). Then (8) becomes

$$
\begin{aligned}
& \frac{d q_{0}}{d t}=A\left(1-q_{2}\right), \\
& \frac{d q_{1}}{d t}=\sin ^{2} q_{0}, \\
& \frac{d q_{2}}{d t}=\cos q_{0} \sin q_{0},
\end{aligned}
$$

and

$$
H=\frac{1}{2}\left(A\left(1-q_{2}\right)^{2}+\sin ^{2} q_{0}\right)
$$

It is interesting to notice that the first and third equations of this system form a onedegree of freedom Hamiltonian system with the Hamiltonian (9). The level lines of this Hamiltonian (i.e., in fact solutions of this subsystem) are shown in Figure 1.

This observation shows that sub-Riemannian geodesics can be of several different types:

- Type 1. Trivial geodesics, corresponding to the minima of $H$ located at the points $q_{2}=1, q_{0}=\pi k, k \in \mathbb{Z}$. For these geodesics $H=0$ and hence therefore there is no motion.

- Type 2. The geodesics corresponding to the saddle equilibria of $H$ located at points $q_{2}=1, q_{0}=\pi / 2+\pi k, k \in \mathbb{Z}$. For such geodesics, $q_{0}$ and $q_{2}$ remain constant, but $q_{1}(t)=t$. Geometrically, these are straight lines.

- Type 3. Geodesics that are periodic in variables $q_{0}$ and $q_{2}$, whereas $q_{1}(t)$ is strictly increasing. They correspond to closed level lines $\{H=h \leqslant 1 / 2\}$ shown in Figure 1. From the view point of the dynamics in $\mathbb{R}^{3}\left(q_{0}, q_{1}, q_{2}\right)$, they are located on invariant cylinders. 


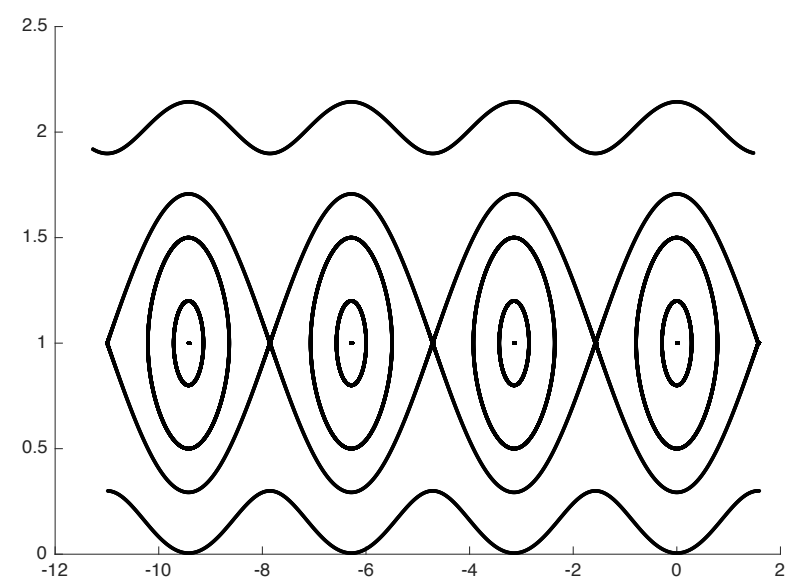

Figure 1: Level lines of $H\left(q_{0}, q_{2}\right)$

- Type 4. Geodesics that correspond to non-closed level lines of $\{H=h \geqslant 1 / 2\}$ shown in Figure 1.

- Type 5. Geodesics that corresponds to separatrices connecting two saddle equilibrium points in Figure 1. The are located at the critical level $\{H=1 / 2\}$. From the view point of the dynamics in $\mathbb{R}^{3}\left(q_{0}, q_{1}, q_{2}\right)$, they asymptotically approach two "critical" geodesics of type 2 as $t \rightarrow \pm \infty$.

Typical geodesics of types 3,4 and 5 are shown in Figure 2 in projection to the coordinate plane $\mathbb{R}\left(q_{1}, q_{2}\right)$.

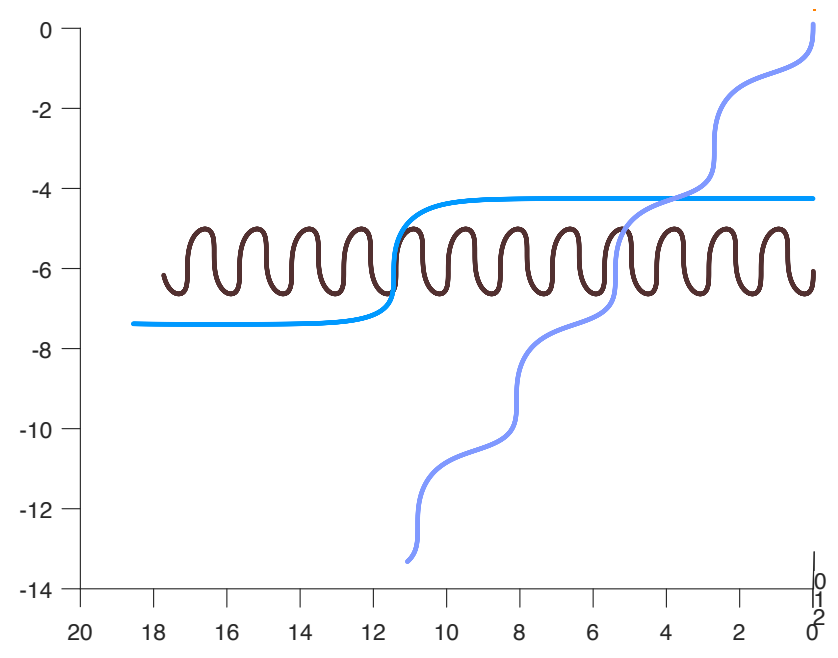

Figure 2: Three types of sub-Riemannian geodesics. 
Locally, system of ODEs (8) can be easily solved in quadratures. For instance, if we consider $\tau=q_{0}$ as a new parameter on geodesics (8), then we have $q_{0}(\tau)=\tau, \quad q_{2}(\tau)=$ $1 \pm \sqrt{\frac{1}{A}\left(2 h-\sin ^{2} \tau\right)}$, where $h$ is a constant of integration. Here we simply use the fact that in terms of $q_{0}$ and $q_{2}$, the solutions coincides with the levels of the Hamiltonian (9), i.e., satisfy the relation $\frac{1}{2}\left(A\left(1-q_{2}(\tau)\right)^{2}+\sin ^{2} q_{0}(\tau)\right)=h$. To recover $q_{1}(\tau)$, it remains to solve the equation

$$
\frac{d q_{1}}{d \tau}=\frac{d q_{1}}{d t} \frac{d t}{d \tau}=\frac{d q_{1}}{d t}\left(\frac{d q_{0}}{d t}\right)^{-1}=\frac{\sin ^{2} q_{0}(\tau)}{A\left(1-q_{2}(\tau)\right)}=\frac{\sin ^{2} \tau}{\sqrt{A\left(2 h-\sin ^{2} \tau\right)}}
$$

so that finally we get the following parametric equation for sub-Riemannian geodesics (with the fixed values of integrals $F_{0}, F_{1}$ and $F_{2}$ )

$$
q_{0}(\tau)=\tau, \quad q_{1}(\tau)=\int \frac{\sin ^{2} \tau d \tau}{\sqrt{A\left(2 h-\sin ^{2} \tau\right)}}, \quad q_{2}(\tau)=1 \pm \sqrt{\frac{1}{A}\left(2 h-\sin ^{2} \tau\right)} .
$$

\section{References}

[1] Arnold, V. I., Mathematical methods of classical mechanics Springer-Verlag, New York, 1989

[2] Barrett, D., Biggs, R., Remsing, C. and Rossi, O., Invariant non-holonomic Riemannian structures on three-dimensional Lie groups. Jour. of Geometric Mechanics 2016, $8(2): 139-167$.

[3] Berestovskii, V. N. and Zubareva, I. A. Locally isometric coverings of the Lie group $S O_{0}(2,1)$ with special sub-Riemannian metric. (Russian) Mat. Sb. 207 (2016), no. 9, 35-56; translation in Sb. Math. 207 (2016), no. 9-10, 1215-1235

[4] Beschastnyi, I. Yu. and Sachkov, Yu. L., Geodesics in the sub-Riemannian problem on the group $S O(3)$. (Russian) Mat. Sb. 207 (2016), no. 7, 29-56; translation in Sb. Math. 207 (2016), no. 7-8, 915-941.

[5] Bizyaev, I. A., Borisov, A. V., Kilin, A. A. and Mamaev, I. S., Integrability and Nonintegrability of Sub-Riemannian Geodesic Flows on Carnot Groups. Regular and Chaotic Dynamics, 2016, vol. 21, no. 6, pp. 759-774.

[6] Bolsinov, A.V. and Taimanov, I.A., Integrable geodesic flows with positive topological entropy. Invent. Math. 140 (2000), 639-650.

[7] Bolsinov, A. V. and Taimanov, I. A., Integrable geodesic flows on suspensions of automorphisms of tori. (Russian) Tr. Mat. Inst. Steklova 231 (2000), Din. Sist., Avtom. i Beskon. Gruppy, 46-63; translation in Proc. Steklov Inst. Math. 2000, no. 4 (231), $42-58$

[8] Bolsinov, A. V. and Jovanovic, B., Integrable geodesic flows on Riemannian manifolds: construction and obstructions. Contemporary geometry and related topics, 57103, World Sci. Publ., River Edge, NJ, 2004. 
[9] Borisov, A. V. and Mamaev, I. S., Rigid body dynamics. De Gruyter Studies in Mathematical Physics, 52. De Gruyter, Berlin; Higher Education Press, [Beijing], 2019. vii +520 pp.

[10] Borisov, A. V. and Mamaev, I. S., Rigid body dynamics in non-Euclidean spaces. Russ. J. Math. Phys. 23 (2016), no. 4, 431-454.

[11] Borisov. A. V., Kilin A. A. and Mamaev, I. S., Hamiltonicity and integrability of the Suslov problem, Regular and Chaotic Dynamics, 2011, vol. 16, no. 1-2, pp. 104-116.

[12] Butler, L., A new class of homogeneous manifolds with Liouville-integrable geodesic flows. C. R. Math. Acad. Sci. Soc. R. Can. 21, No.4, 127-131 (1999).

[13] Fisher D.J., Gray, R.J. and Hydon, P.E., Automorphisms of real Lie algebras of dimension five or less. J. Phys. A 46 (2013), no. 22, 225204, 18 pp.

[14] Harvey, A., Automorphisms of the Bianchi model Lie groups. Journal of Mathematical Physics 20, 251 (1979).

[15] Konyaev, A.Yu., Classification of Lie algebras with generic orbits of dimension 2 in the coadjoint representation, Sb. Math., 205:1 (2014), 45-62

[16] Kruglikov, B. S., Vollmer A. and Lukes-Gerakopoulos, G., On integrability of certain rank 2 sub-Riemannian structures. Regular and Chaotic Dynamics, 2017, vol. 22, no. 5, pp. 502-519.

[17] Lokutsievskii, L. V. and Sachkov, Yu. L., Liouville nonintegrability of sub-Riemannian problems on depth 4 free Carnot groups. (Russian) Dokl. Akad. Nauk 474 (2017), no. 1, 19-21; translation in Dokl. Math. 95 (2017), no. 3, 211-213

[18] Lokutsievskii, L. V. and Sachkov, Yu. L., On the Liouville integrability of subRiemannian problems on Carnot groups of step 4 and higher. (Russian) Mat. Sb. 209 (2018), no. 5, 74-119; translation in Sb. Math. 209 (2018), no. 5, 672-713

[19] Marenitch, V. Geodesic lines in $\widetilde{S L_{2}(R)}$ and Sol. Novi Sad J. Math. 38 (2008), no. 2, 91-104.

[20] Mashtakov, A. P. and Sachkov, Yu. L., Superintegrability of left-invariant subRiemannian structures on unimodular three-dimensional Lie groups. Translation of Differ. Uravn. 51 (2015), no. 11, 1482-1488. Differ. Equ. 51 (2015), no. 11, 1476-1483.

[21] Mashtakov, A. P. and Sachkov, Yu. L., Integrability of left-invariant sub-Riemannian structures on the special linear group $S L_{2}(R)$. Translation of Differ. Uravn. 50 (2014), no. 11, 1541-1547. Differ. Equ. 50 (2014), no. 11, 1541-1547.

[22] Mielke, A., Finite elastoplasticity, Lie groups and geodesics on $S L(d)$ : In the book Geometry, Mechanics and Dynamics. Springer, New York, 2002, 61-90.

[23] Mishchenko, A. S. and Fomenko, A. T., Euler equations on finite-dimensional Lie groups, Math. USSR-Izv., 12, No. 2, 371-389 (1978). 
[24] Mishchenko, A. S. and Fomenko, A. T. Generalized Liouville method of integration of Hamiltonian systems Funktsional. Anal. i Prilozhen. 12 (2) (1978), 46-56, [in Russian]. English translation: Functional Anal. Appl. 12 (1978), 113-121.

[25] Mishchenko, A. S. and Fomenko, A. T., Integration of hamiltonian systems with non-commutative symmetries, Tr. Semin. Vektorn. Tenzorn. Anal. 20 (1981), 5-54. (Russian)

[26] Patera, J., Sharp, R. T., Winternitz, P., and Zassenhaus, H., Invariants of real low dimension Lie algebras. Journal of Mathematical Physics 17 (1976), 986-994.

[27] Sadetov, S. T., A proof of the Mishchenko-Fomenko conjecture, Dokl. Akad. Nauk 397 (2004), no. 6, 751-754.

[28] Whittaker, E. T., A Treatise on the Analytical Dynamics of Particles and Rigid Bodies (Cambridge Mathematical Library) 4th Edition Cambridge University Press; 4 edition (February 24, 1989). 